\title{
BMC Biophysics reviewer acknowledgement 2015
}

Julia Simundza

\section{Contributing reviewers}

The editors of BMC Biophysics would like to thank all our reviewers who have contributed to the journal in Volume 8 (2015).

\author{
Nadav Amdursky \\ UK
}

Nurit Ashkenasy

Israel

Marc Baaden

France

Andrew Battle

Australia

Timo Betz

Germany

Roland Brandt

Germany

Robert Brewster

USA

Oscar Domenech

Spain

Alexander Dunn

USA

Paolo Facci

Italy

Reza Farhadifar

USA

Klemens Fellner

Austria
Nir Gov
Israel

Ingo Greger

UK

Chen $\mathrm{Hu}$

China

Mohan Joshi

USA

Pavel Jungwirth

Czech Republic

Werner Kremer

Germany

Douglas Laurents

Spain

Timothy Lezon

USA

Benzhuo Lu

China

Alexander Mackerell

USA

Agostino Migliore

USA

Namiko Mitarai

Denmark

\section{Sorin Mitran \\ USA}

Anders Olofsson

Sweden

Vytautas Petrauskas

Lithuania

Miquel Pons

Spain

Mathis Riehle

UK

Arun Sampathkumar

USA

Gudrun Schappacher-Tilp

Austria

Helmut Schiessel

Netherlands

Michael Schlierf

Germany

Terence Strick

France

Piotr Szymczak

Poland

Joanna Trylska

Poland 
M. Ubbink

Netherlands

Stephan Uphoff

UK
Ady Vaknin

Israel

Sivanandam Veeramuthu USA
Chung Wong

USA 